\title{
Growth Failure in Children with Systemic Juvenile Idiopathic Arthritis and Prolonged Inflammation despite Treatment with Biologicals: Late Normalization of Height by Combined Hormonal Therapies
}

\author{
Francis de Zegher $^{\mathrm{a}} \quad$ Nele Reynaert ${ }^{\mathrm{a}}$ Lien De Somer $^{\mathrm{b}} \quad$ Carine Wouters $^{\mathrm{b}}$ \\ Mathieu Roelants ${ }^{c}$ \\ a Pediatric Endocrinology, University Hospital Gasthuisberg, Leuven, Belgium; bediatric Rheumatology, University Hospital \\ Gasthuisberg, Leuven, Belgium; ' ${ }^{\mathrm{D}}$ epartment of Environment and Health, University of Leuven, Leuven, Belgium
}

\section{Established Facts}

- Biologicals targeting the interleukin (IL)-1 $\beta$ or IL-6 pathway are increasingly used to treat children with systemic juvenile idiopathic arthritis (sJIA); up to 1 in 3 children with sJIA continues to present inflammatory activity, to require glucocorticoid treatment, and to grow slowly for years, and may end up with a short adult stature.

- The administration of growth hormone (GH) to sJIA children with ongoing glucocorticoid-treated inflammation can result in an acceleration of height velocity but is accompanied by severe insulin resistance and may not be an effective way of normalizing adult stature.

\section{Novel Insights}

- We report long-term, proof-of-concept evidence that the adult height of short sJIA children can be normalized - albeit late - with a combined strategy wherein (i) pubertal development is postponed (with triptorelin) either until a minimum height is reached or until prepubertal height gain is exhausted, and (ii) growth-promoting treatment (with GH) is initiated when inflammation is under control, when glucocorticoid treatment is no longer needed, and when endogenous activity in the adreno-corticotropic axis is still recovering, thus in a phase of relative glucocorticoid deficiency. This approach may also be applicable to other conditions with prolonged inflammation and/or glucocorticoid excess.

\section{Keywords}

Systemic juvenile idiopathic arthritis · Short stature ·

Growth hormone · Puberty

\begin{abstract}
Background: Biologicals targeting the interleukin (IL)- $1 \beta$ or IL-6 pathway are becoming prime choices for the treatment of children with systemic juvenile idiopathic arthritis (sJlA). Up to 1 in $3 \mathrm{~s}$ اA children receiving such treatment continues to have inflammatory activity and to require supra-physiological glucocorticoid doses which may reduce growth ve-
\end{abstract}

\section{KARGER}

(C) 2018 S. Karger AG, Basel

E-Mail karger@karger.com

www.karger.com/hrp
Francis de Zegher, $\mathrm{MD}, \mathrm{PhD}$

Pediatric Endocrinology

University Hospital Gasthuisberg

BE-3000 Leuven (Belgium)

E-Mail francis.dezegher@uzleuven.be 
locity for years and may lead to an extremely short stature for age, if not for life. Currently, there is no long-term proposal to normalize the adult height of these children with sJIA. Methods and Results: We present long-term (up to 10 years), proof-of-concept evidence that the adult stature and adipose body composition of short sاJA children can be normalized with a hormonal combination strategy: (i) pubertal onset is postponed with a gonadotropin-releasing hormone analog (triptorelin) until a minimum height is reached, or until prepubertal growth is exhausted, and (ii) height gain is promoted with growth hormone ( $\approx 50 \mu \mathrm{g} / \mathrm{kg} /$ day), once inflammation is under control and high glucocorticoid doses are no longer needed. The latter treatment takes advantage of the window of relative glucocorticoid deficiency, which is known to open after prolonged glucocorticoid administration, and to be uniquely favorable to height gain. Conclusion: A long-term combination of biological and hormonal treatments for short sJIA children can be guided by a simple concept that involves (i) postponement of pubertal development and (ii) growth-promoting therapy after the episodes of major inflammation and high-dose glucocorticoid treatment. Limited long-term experience in short sJIA children suggests that this strategy leads consistently - albeit late - to a normal adult stature.

(c) 2018 S. Karger AG, Basel

\section{Background}

Biologicals targeting the interleukin (IL) $-1 \beta$ or IL-6 pathway are becoming prime choices to treat children with systemic juvenile idiopathic arthritis (sJIA) $[1,2]$. The majority of children with sJIA respond favorably to such an intervention: they display less inflammatory activity, need less glucocorticoids, and tend to normalize their growth velocity so that their height centiles either stabilize [3, 4] or partly return to pre-sJIA levels [5]. However, up to 1 in 3 sJIA children under biological treatment continues to have inflammatory activity and to require supra-physiological glucocorticoid doses; these children grow slowly for years, thus ending up with a short stature - which may be extreme - by late childhood $[6,7]$. The administration of growth hormone $(\mathrm{GH})$ to sJIA children with ongoing glucocorticoid-treated inflammation can result in an acceleration of height velocity; however, this strategy is a relatively ineffective way of increasing adult stature and may be accompanied by severe insulin resistance with a risk for impaired glucose tolerance, and even for diabetes [8-10].

\section{General Concept}

We present long-term, proof-of-concept evidence that adult stature of short sJIA children can be normalized albeit late - with a hormonal combination strategy wherein (i) pubertal development is postponed either until a minimum height is reached [11] or until prepubertal height gain is exhausted, and (ii) growth-promoting treatment is only initiated once the inflammation is under control, and thus once high doses of glucocorticoids are no longer needed. This strategy fits with the suggestion to reduce the height deficit of short sJIA children prior to the onset of puberty [12], and with the observation that inflammatory disease activity tends to attenuate the growth-promoting effect of GH [9]. This strategy should be clearly explained to - and fully endorsed by - the patients and their parents, prior to its implementation and during the entire treatment sequence; our experience is that informed parents and children prioritize consistently a more normal (taller) adult stature over a more normal (less delayed) pubertal timing.

\section{Biologicals and the Onset of Puberty: Normal Age versus Normal Height}

Biological-entrained reduction of generalized inflammation in late childhood can be accompanied by onset of pubertal development whose timing may be normal (and even late-normal) for chronological age but is often early for body height. Short children, who start puberty at a normal age but at a low height, are known to end up with a short adult stature [11]. Accordingly, we initiate a concomitant intervention with a gonadotropin-releasing hormone analog (triptorelin) in such sJIA children, thereby postponing their pubertal development until a more normal late-prepubertal height is reached.

For example, the girl, whose longitudinal course is shown in Figure 1, did start with breast development at age 12.3 years - within 4 months after initiating IL- $1 \beta$ blockade - at a height of $110 \mathrm{~cm}$; she received triptorelin for 3.5 years (between ages 12.5 and 16.0 years), thus reaching a more normal late-prepubertal height (136 $\mathrm{cm}$ ), before initiating a pubertal growth spurt toward an adult stature comparable to that of her mother. The vertebral (L1-L4) bone mineral density (BMD) Z-scores of this girl were low (approximately -3.0 between 8 and 12 years of age, and approximately -2.4 between 14 and 16 years) but normal $(+0.3)$ at the age of 23 years, thus $>5$ years after stopping all interventions. 
Fig. 1. Height velocity, height $Z$-score, body composition, and disease activity score in a sJIA girl (a) and a sJIA boy (b) before and after the start of treatment with biologicals, triptorelin, and growth hormone. Both children experienced severe growth faltering that stabilized under biologicals, but catch-up growth did not occur prior to additional treatment with growth hormone. Triptorelin treatment was initiated at onset of puberty (B2 at a height of $110 \mathrm{~cm}$ in the girl; G2 and testicular volume of $8 \mathrm{~mL}$ at a height of $127.5 \mathrm{~cm}$ in the boy); pubertal development resumed upon withdrawal of triptorelin treatment. Although their BMD $Z$-scores were below -2 up to the age of 16 years, these children did not experience any vertebral or other fracture. The fat/lean mass indicator illustrates that body composition remained adipose under biologicals, and that additional growth hormone treatment prioritized the gain of lean mass, thereby normalizing body composition. In the girl, the sharp rise of body adiposity between the ages of 10 and 11 years was observed during treatment with thalidomide. The grey areas represent estimates of normal ranges (centiles 3 and 97 for height velocity; centiles 2 and 85 for fat/ lean mass) for sex and chronological age; in the disease activity panel, the darker the shade of grey, the higher the disease activity score (see Appendix: Methods). DXA, dual X-ray absorptiometry.

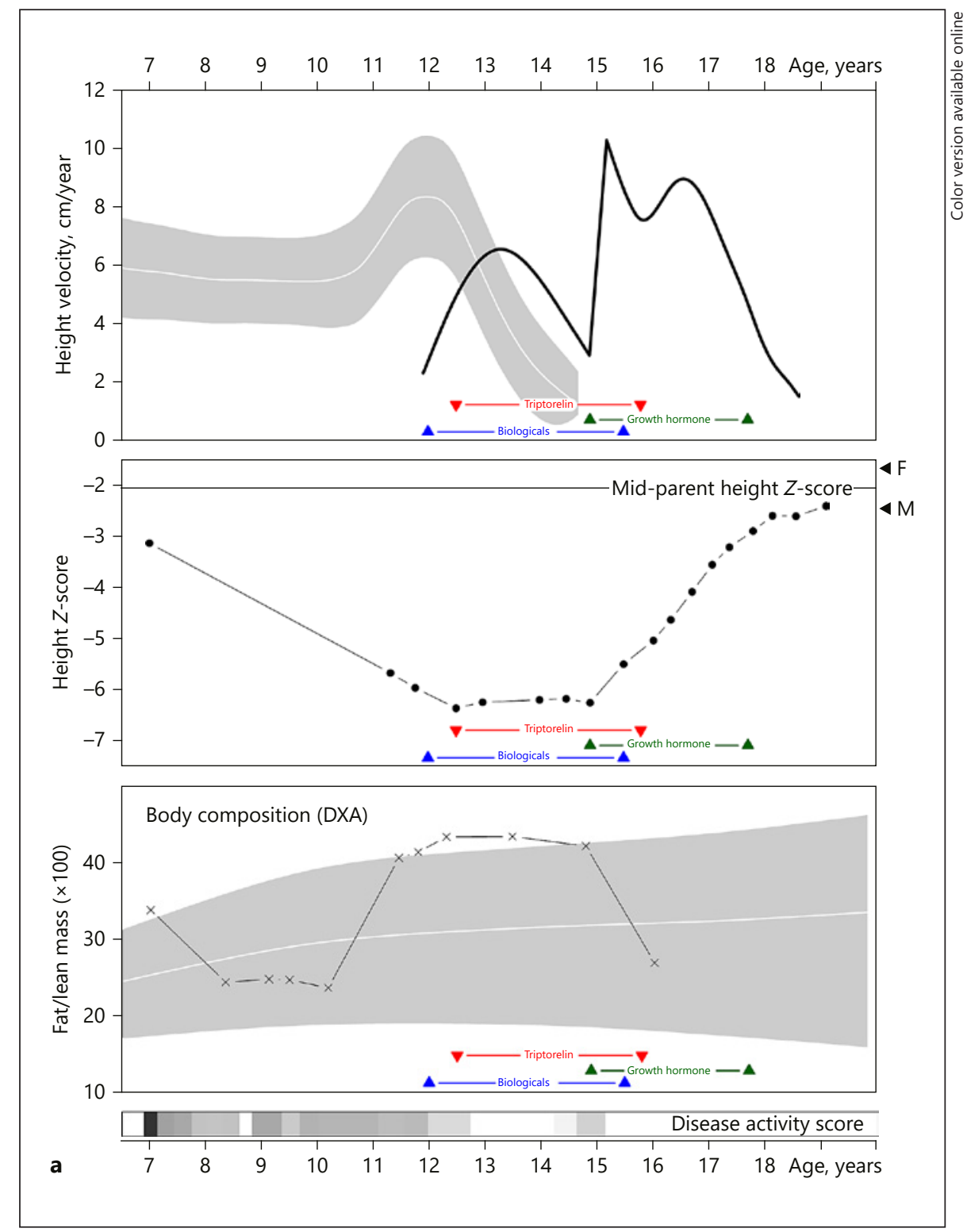

(Figure continued on next page.)
A limitation of the present report is that we did not perform repetitive screenings for asymptomatic vertebral fractures; future studies will clarify whether a delayed gain of BMD is a cause of concern.

\section{Biologicals and Height Gain: Without GH versus with GH}

When long-term glucocorticoid treatment with supraphysiological doses can finally be lowered into the infraphysiological range, then it may take years for the hypo- thalamo-pituitary components of the adreno-corticotropic axis to return to a fully functional state [13]. Such a slow recovery - perhaps the slowest in the human endocrine system - opens a prolonged time window of relative glucocorticoid deficiency, which has long been known to be uniquely favorable to height gain [14-16].

In the absence of exogenous GH (Fig. 2a, b, left and middle sections), our findings confirm that the initiation of a biological treatment and the concomitant reduction of glucocorticoid doses tend to be followed by modest increments of height velocity [3-5]. However, these increments do not result in a frank "catch-up," when analyses 


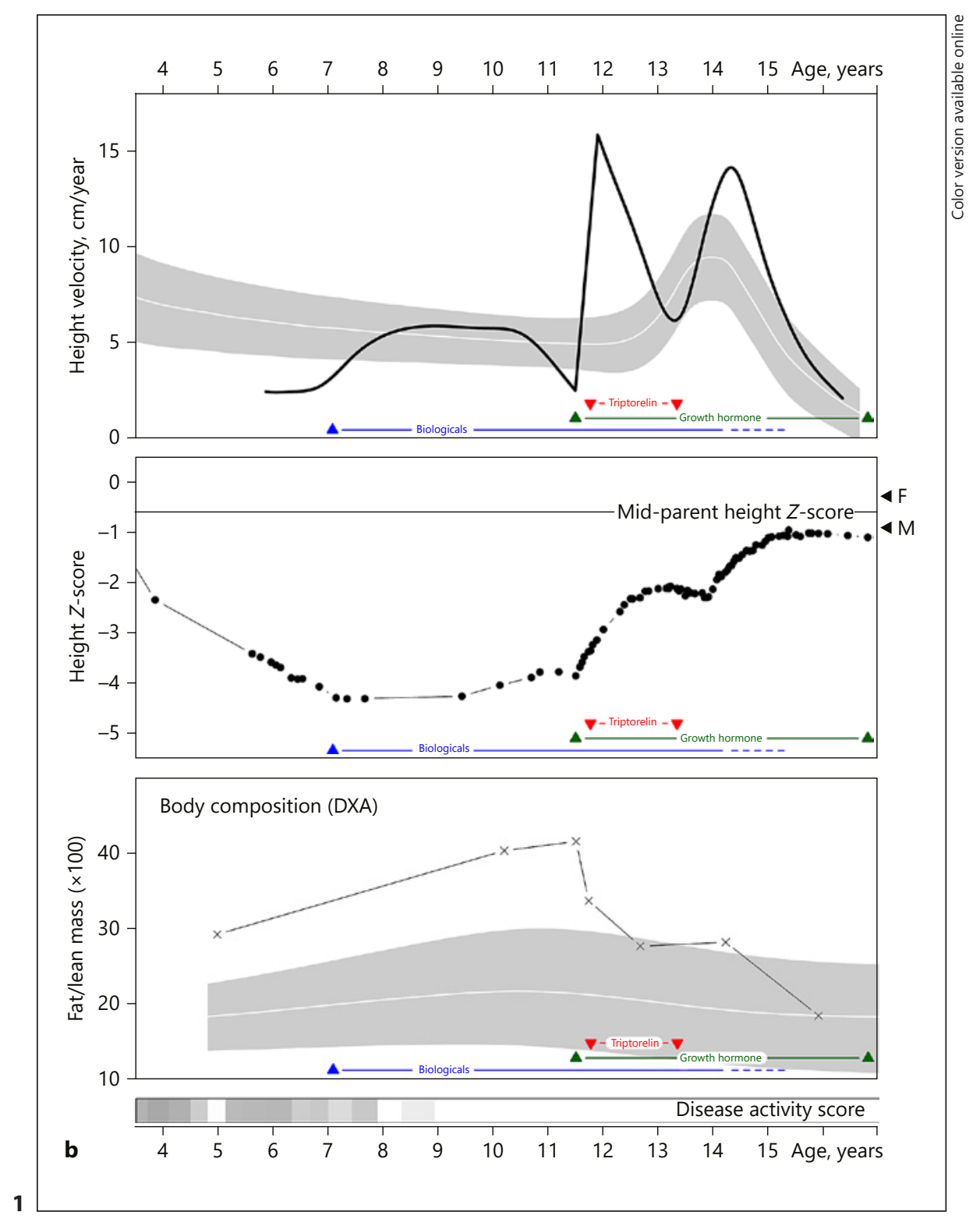

are restricted to children who stay prepubertal, either spontaneously or pharmacologically (with triptorelin). Longitudinal body-composition assessments were performed in a few of these sJIA children, and they showed that treatment with a biological fails to attenuate body adiposity toward normal (Fig. 1, lower panels).

When GH therapy $(\approx 50 \mu \mathrm{g} / \mathrm{kg} /$ day $)$ was added in an on-biological, post-glucocorticoid, low-inflammatory phase, then marked prepubertal growth accelerations were observed, comparable to the "catch-up" responses of GH-deficient children (Fig. 2a, b, right section). Such $\mathrm{GH}$ interventions were also accompanied by striking re- ductions of body adiposity toward normal (Fig. 1, lower panels). The mechanisms underpinning these impressive responses remain to be uncovered. In children with severe JIA, the prevalence of GH deficiency may be as high as $18 \%$ [9]. In addition, since the GH receptor is a cytokine receptor, it is possible that an abundant presence of IL- $1 \beta$ or IL- 6 reduces GH activity and, conversely, that a blockade of such IL- $1 \beta$ or IL- 6 interference tends to normalize the actions of $\mathrm{GH}$.

The limitations of the present report include the absence of evidence related to insulin sensitivity on $\mathrm{GH}$ treatment, and the low number of IGF-1 assessments, 
Fig. 2. Annualized height velocity (a) and growth response (b) of sJIA children the year before and the year after starting treatment with a biological and then also with growth hormone $(\mathrm{GH})$. The results are from the first 5 short children who could be followed over several years, and who remained prepubertal during these growth phases, either spontaneously or pharmacologically (with triptorelin); these children did not experience any vertebral or other fracture. Growth responses are expressed in height velocity $Z$-scores relative to the height velocities observed during the first year of GH treatment in late-prepubertal children with GH deficiency (GHD). The lower boundary of the response range of GHD children ( $Z$-score -2$)$ is close to the mean growth velocity in the reference population and can thus be considered as the lower boundary of catch-up growth. When growth responses are not confounded by puberty, then treatment with a biological is often accompanied by a growth acceleration in sJIA children, but rarely by true "catch-up" growth. The latter can be achieved by adding GH. Apparent paradoxes about the absence $[3,4]$ versus presence [5] of "catch-up" growth after starting a biological treatment can thus be explained by the confounding influence of early-pubertal growth accelerations. It remains to be studied whether sJIA children under prolonged biological treatment may develop GHD.
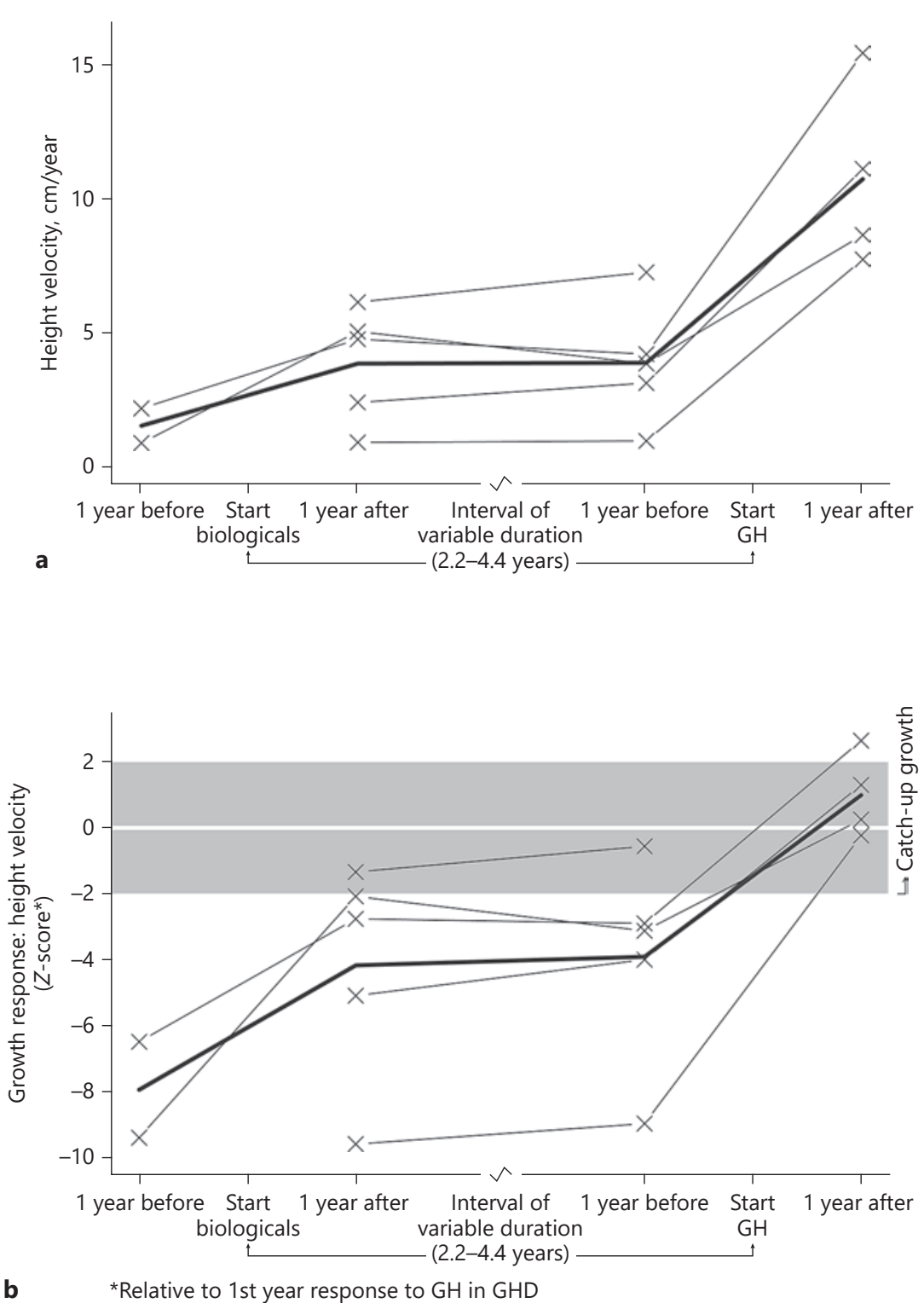

b which disclosed circulating concentrations mostly in the 250-400 ng/mL range during prepubertal GH treatment, and in the $450-700 \mathrm{ng} / \mathrm{mL}$ range during pubertal $\mathrm{GH}$ treatment.

\section{Conclusion}

Parallel, sequential, intermittent, and longer-term combinations of biological and hormonal treatments for short sJIA children can be guided by a simple concept and

Treatment of Growth Failure in Children with sJIA can be individualized, depending on factors such as sJIA timing and severity, the responsiveness to biologicals, the need for glucocorticoids, and the frequency and duration of sJIA flares. There is a need for clinical trials in this orphan field but, given the abovementioned factors, the design and completion of such longer-term studies may prove not to be an easy task.

Our long-term experience with short stature in contemporary sJIA children is too limited to allow for recommendations, but it may suffice to allow even the shortest sJIA children to maintain the hope for a normal adult 
stature. We view a normal adult height as a distant target that can be reached by any sJIA child, provided body growth is gently guided through the changing circumstances. Such guidance may require a lot of patience, and safe arrival at a normal adult stature may be late.

\section{Appendix: Methods}

Height, weight, absorptiometry-derived body fat and lean mass, vertebral BMD $Z$-scores, height of the parents, and medical history (including inflammatory activity scores and treatment modalities) were extracted from the clinical records of 5 extremely short children with sJIA who were treated with a biological $[1,2]$ and were followed jointly in the Pediatric Rheumatology and Pediatric Endocrinology Units at the University Hospital Gasthuisberg in Leuven, Belgium. Biologics were administered within clinical studies requiring informed consent $[1,2]$, subsequently via compassionate use, and ultimately as conventional therapy; triptorelin (Decapeptyl, Ipsen) was administered within a compassionate-use program, and rhGH (Genotonorm, Pfizer) within a medical-need program requiring informed consent.

In Figure 1, height velocities were calculated as height differences between 2 measurements divided by the time interval, and are expressed in $\mathrm{cm} /$ year. All possible combinations of measurement occasions were taken into consideration, but only those within 6 months of a 1-year interval were retained for analysis. Because the start of treatment with GH marks a sudden change in growth pattern, the height velocities were calculated separately for measurements before versus after the start of this treatment. Velocity estimates were spline smoothed to indicate observed trends, and plotted with corresponding sex-matched centiles 3, 50, and 97 of peak height velocity-centered annual growth velocities [17] in the background.
Height was converted into $Z$-scores according to the local growth reference [18]. Mid-parent height $Z$-score was calculated as mean height $Z$-score of both parents multiplied by 0.72 [19].

An index of $100 \times$ fat mass/lean mass (by dual X-ray absorptiometry) was used as a measure of body adiposity; the reference ranges are derived from reference values for percentage body fat $(\% \mathrm{BF})$ in Caucasian children $[20]$ as $100 \times \% \mathrm{BF} /(1-\% \mathrm{BF})$, using centiles 2 and 85 as boundaries. This formula includes bone mass in the denominator which results in a downward bias of approximately 0.5 units for the lower range and 2 units for the upper range.

Inflammatory activity is indicated by the composite Juvenile Arthritis Disease Activity Score (JADAS) using an active joint count in 71 joints [21]; these scores are presented as a monochrome heatmap based on the logarithmically transformed JADAS, wherein darker zones indicate more disease activity (the maximum observed score was 78) and white zones indicate no disease activity (score 0 ). The scores were logarithmically transformed in order to discriminate consecutive disease phases with a relatively low score.

In Figure 2, annualized height velocity ( 1 year before and 1 year after the start of treatment with biologicals and GH) is based on the measurements at treatment start, and those nearest to a whole year before or after the start of treatment (range 0.6-1.4 years); height velocity $Z$-scores were calculated using a growth velocity reference for the first year of treatment with $\mathrm{GH}$ in children with GH deficiency [22].

\section{Disclosure Statement}

The authors have nothing to disclose.

\section{References}

1 Ruperto N, Brunner HI, Quartier P, Constantin T, Wulffraat N, Horneff G et al.; PRINTO; PRCSG. Two randomized trials of canakinumab in systemic juvenile idiopathic arthritis. N Engl J Med. 2012 Dec;367(25):2396-406.

2 De Benedetti F, Brunner HI, Ruperto N, Kenwright A, Wright S, Calvo I et al.; PRINTO; PRCSG. Randomized trial of tocilizumab in systemic juvenile idiopathic arthritis. N Engl J Med. 2012 Dec;367(25):2385-95.

3 Yokota S, Imagawa T, Mori M, Miyamae T, Takei S, Iwata N et al. Long-term treatment of systemic juvenile idiopathic arthritis with tocilizumab: results of an open-label extension study in Japan. Ann Rheum Dis. 2013 Apr; 72(4):627-8.
4 Yokota S, Imagawa T, Mori M, Miyamae T, Takei S, Iwata $\mathrm{N}$ et al. Longterm safety and effectiveness of the anti-interleukin 6 receptor monoclonal antibody tocilizumab in patients with systemic juvenile idiopathic arthritis in Japan. J Rheumatol. 2014 Apr;41(4):759-67.

5 De Benedetti F, Brunner H, Ruperto N, Schneider R, Xavier R, Allen R et al.; Paediatric Rheumatology International Trials Organisation and the Pediatric Rheumatology Collaborative Study Group. Catch-up growth during tocilizumab therapy for systemic juvenile idiopathic arthritis: results from a phase III trial. Arthritis Rheumatol. 2015 Mar;67(3): 840-8.

6 Uettwiller F, Perlbarg J, Pinto G, BaderMeunier B, Mouy R, Compeyrot-Lacassagne $S$ et al. Effect of biologic treatments on growth in children with juvenile idiopathic arthritis. J Rheumatol. 2014 Jan;41(1):128-35.
7 Klotsche J, Raab A, Niewerth M, Sengler C, Ganser G, Kallinich T et al. Outcome and trends in treatment of systemic juvenile idiopathic arthritis in the German National Pediatric Rheumatologic Database, 2000-2013. Arthritis Rheumatol. 2016 Dec;68(12):302334.

8 Simon D, Prieur AM, Quartier P, Charles Ruiz J, Czernichow P. Early recombinant human growth hormone treatment in glucocorticoid-treated children with juvenile idiopathic arthritis: a 3-year randomized study. J Clin Endocrinol Metab. 2007 Jul;92(7):256773.

9 Bechtold S, Ripperger P, Dalla Pozza R, Bonfig W, Häfner R, Michels H et al. Growth hormone increases final height in patients with juvenile idiopathic arthritis: data from a randomized controlled study. J Clin Endocrinol Metab. 2007 Aug;92(8):3013-8. 
10 Wong SC, Dobie R, Altowati MA, Werther GA, Farquharson C, Ahmed SF. Growth and the growth hormone-insulin like growth factor 1 axis in children with chronic inflammation: current evidence, gaps in knowledge, and future directions. Endocr Rev. 2016 Feb; 37(1):62-110.

11 van der Steen M, Lem AJ, van der Kaay DC, Bakker-van Waarde WM, van der Hulst FJ, Neijens FS et al. Metabolic health in short children born small for gestational age treated with growth hormone and gonadotropin-releasing hormone analog: results of a randomized, dose-response trial. J Clin Endocrinol Metab. 2015 Oct;100(10):3725-34.

12 Bechtold S, Beyerlein A, Ripperger P, Roeb J, Dalla Pozza R, Häfner R et al. Total pubertal growth in patients with juvenile idiopathic arthritis treated with growth hormone: analysis of a single center. Growth Horm IGF Res. 2012 Oct;22(5):180-5.

13 Huber BM, Bolt IB, Sauvain MJ, Flück CE. Adrenal insufficiency after glucocorticoid withdrawal in children with rheumatic diseases. Acta Paediatr. 2010 Dec;99(12):188993.
14 Shepard TH, Landing BH, Mason DG. Familial Addison's disease; case reports of two sisters with corticoid deficiency unassociated with hypoaldosteronism. AMA J Dis Child. 1959 Feb;97(2):154-62.

15 Migeon CJ, Kenny EM, Kowarski A, Snipes CA, Spaulding JS, Finkelstein JW et al. The syndrome of congenital adrenocortical unresponsiveness to ACTH. Report of six cases. Pediatr Res. 1968 Nov;2(6):501-13.

16 Elias LL, Huebner A, Metherell LA, Canas A, Warne GL, Bitti ML et al. Tall stature in familial glucocorticoid deficiency. Clin Endocrinol (Oxf). 2000 Oct;53(4):423-30.

17 Tanner JM, Whitehouse RH, Takaishi M. Standards from birth to maturity for height, weight, height velocity, and weight velocity: British children, 1965. II. Arch Dis Child. 1966 Dec;41(220):613-35.

18 Roelants M, Hauspie R, Hoppenbrouwers K. References for growth and pubertal development from birth to 21 years in Flanders, Belgium. Ann Hum Biol. 2009 Nov-Dec;36(6): 680-94.
19 Hermanussen M, Cole J. The calculation of target height reconsidered. Horm Res. 2003; 59(4):180-3.

20 McCarthy HD, Cole TJ, Fry T, Jebb SA, Prentice AM. Body fat reference curves for children. Int J Obes. 2006 Apr;30(4):598602.

21 Consolaro A, Ruperto N, Bazso A, Pistorio A, Magni-Manzoni S, Filocamo G et al.; Paediatric Rheumatology International Trials Organisation. Development and validation of a composite disease activity score for juvenile idiopathic arthritis. Arthritis Rheum. 2009 May;61(5):658-66.

22 Straetemans S, Roelants M, Thomas M, Rooman R, De Schepper J. Reference curve for the first-year growth response to growth hormone treatment in prepubertal children with idiopathic growth hormone deficiency: validation of the KIGS first-year growth response curve using the Belgian Register for the Study of Growth and Puberty Problems. Horm Res Paediatr. 2014; 81(5):343-9. 